This is an author produced version of a paper published in Physical Chemistry Chemical Physics.

This paper has been peer-reviewed but may not include the final publisher proof-corrections or pagination.

Citation for the published paper:

Josephina Werner, Jan Julin, Maryam Dalirian, Nønne L. Prisle, Gunnar Öhrwall, Ingmar Persson, Olle Björneholm, and Ilona Riipinen. (2014)

Succinic acid in aqueous solution: connecting microscopic surface composition and macroscopic surface tension. Physical Chemistry Chemical Physics. Volume: 16, Number: 39, pp 21486-21495. http://dx.doi.org/10.1039/c4cp02776k.

Access to the published version may require journal subscription. Published with permission from: Royal Society of Chemistry.

Epsilon Open Archive http://epsilon.slu.se 


\title{
Succinic acid in aqueous solution: Connecting microscopic surface composition and macroscopic surface tension ${ }^{\dagger}$
}

\author{
Josephina Werner, ${ }^{* a, b}$ Jan Julin, ${ }^{c}$ Maryam Dalirian, ${ }^{c}$ Nønne L. Prisle, ${ }^{d}$ Gunnar Öhrwall,,${ }^{e}$ Ingmar \\ Persson, ${ }^{b}$ Olle Björneholm, ${ }^{a}$ and Ilona Riipinen ${ }^{c, f}$
}

author's preprint $21 / 08-2014$

The water-vapor interface of aqueous solutions of succinic acid, where $\mathrm{pH}$ value and bulk concentration were varied, has been studied using surface sensitive X-ray photoelectron spectroscopy (XPS) and molecular dynamics (MD) simulations. It was found that succinic acid has a considerably higher propensity to reside in the aqueous surface region than its deprotonated form, which is effectively depleted from the surface due to the two strongly hydrated carboxylate groups. From both XPS experiments and MD simulations a strongly increased concentration of the acid form in the surface region compared to the bulk concentration was found and quantified. Detailed analysis of the surface of succinic acid solutions at different bulk concentrations led to the conclusion that succinic acid saturates the aqueous surface at high bulk concentrations. With the aid of MD simulations the thickness of the surface layer could be estimated, which enabled the quantification of surface concentration of succinic acid as multiple of the known bulk concentration. The obtained enrichment factors were successfully used to model surface tension of these binary aqueous solutions using two different models that account for surface enrichment. This underlines the close correlation of increased concentration at the surface relative to the bulk and reduced surface tension of aqueous solutions of succinic acid. The results of this study shed light on the microscopic origin of surface tension, a macroscopic property. Furthermore, the impact of the results from this study on atmospheric modeling is discussed.

\section{Introduction and motivation}

Atmospheric aerosols have a significant effect on the Earth's radiation budget and can therefore affect the climate by either direct scattering of solar radiation or indirectly by changing the properties and lifetime of clouds. ${ }^{1}$ To better understand the impact of atmospheric aerosol particles, a detailed picture of their formation and evolution processes is required.

There is a large variety of organic compounds present in the atmosphere, and their properties or even identities are still very poorly known. ${ }^{2}$ Dicarboxylic acids, such as oxalic, malonic, succinic and glutaric acid, are a group of organic compounds that are known oxidation products of biogenic volatile organic

$0 \dagger$ Electronic Supplementary Information (ESI) available: Acid-base titration of succinic acid and $\mathrm{C} 1 \mathrm{~s}$ PE spectra of succinic acid at $\mathrm{pH} 2.0,4.6$ and 12.9. Furthermore, aspects concerning surface sensitivity of XPS experiments and the derivation of surface enrichment factors are discussed. See DOI $\mathrm{xx} . \mathrm{xxxx} / \mathrm{x} 000000 \mathrm{x} /$

$0^{a}$ Department of Physics and Astronomy, Uppsala University, P.O. Box 516, SE-751 20 Uppsala, Sweden Tel: +4618 471 3561; E-mail: josephina.werner@physics.uu.se

$0^{b}$ Department of Chemistry and Biotechnology, Swedish University of Agricultural Sciences, P.O. Box 7015, SE-750 07 Uppsala, Sweden

$0^{c}$ Department of Applied Environmental Science and Bolin Centre for Climate Research, Stockholm University, SE-106 91 Stockholm, Sweden

$0^{d}$ Department of Physics, Helsinki University , P.O. Box 64, FIN-00014 Helsinki, Finland

$0^{e}$ MAX IV Laboratory, Lund University, P.O. Box 118, SE-221 00 Lund, Sweden

$0{ }^{f}$ Center for Atmospheric Particle Studies (CAPS), Carnegie Mellon University, Pittsburgh, PA 15213, USA compounds in the atmosphere. ${ }^{3}$ These volatile compounds are oxidized and transformed via atmospheric radical and photochemical processes to less volatile compounds, which in turn may, due to their low saturation vapor pressures, contribute to atmospheric aerosol loadings and composition. To understand their role in aerosol formation and cloud activation, various physico-chemical properties, such as saturation vapor pressures, surface tensions, densities and interactions with other atmospheric molecules, such as water or inorganic salts, need to be understood. In this context, extensive experimental investigations on dicarboxylic acids have been reported in the past few years, where particular focus has been on measuring their saturation vapor pressures, surface tensions, densities (as both pure compounds as well as aqueous solutions) and liquid phase activities in sub-saturated aqueous solutions. ${ }^{4-7}$ An issue of high atmospheric relevance is the surface to bulk partitioning of surface active compounds and their ability to alter the surface tension. ${ }^{8-10}$ As they accumulate in the narrow region where the transition between bulk liquid and vapor phase takes place, surface active compounds may modify properties of the surface region. ${ }^{11} \mathrm{~A}$ larger amount of these compounds close to the water-vapor interface can also affect interactions between the condensed phase and the vapor phase. ${ }^{12,13}$

In general, surface tension is a macroscopic feature, as it is the reason why water form spherical droplets to minimize their surface to bulk ratio, while its origin lies in microscopic molecular interactions. In thermodynamic model calculations sur- 
face tension is expressed as the energy change associated with increasing the surface area by filling the surface with fully coordinated bulk atoms. But only the mechanical surface tension, which is the force related to expanding the surface, or in other words by which surface area is created, ${ }^{11}$ can be measured directly. This dualistic definition of a surface of an aqueous solution complicates the understanding of surface tension and its effects.

There is a general need for supplementary microscopic insight to be able to discuss differences between measured surface tension and calculated ones using thermodynamic models. Accurate probing of the interfacial distribution of organic compounds provides further insight on the origin of changes in surface tension and sheds light on prevailing intermolecular interactions. As it is used to model cloud droplet activation and nucleation rates, an uncertainty in surface tension results in an uncertainty in predictions of aerosol-cloud interactions and aerosol formation rates.

XPS is a surface sensitive technique, which is well established and successfully applied for characterization of solids, gases, clusters and liquids. ${ }^{14,15}$ The main advantages of XPS are that both the chemical state and the microscopic spatial distribution of the compounds can be probed directly. Utilizing this technique together with a liquid micro-jet setup, measured surface compositions of succinic acid in aqueous solution $\left(\mathrm{SuccH}_{2}\right)$ for various concentrations and $\mathrm{pH}$ values are reported in this work. Succinic acid was chosen as a model compound representing the group of water soluble organic compounds which are known to be present in atmospheric aerosols and which due to their low vapor pressure possibly contribute to aerosol formation and growth processes. The results from surface sensitive XPS experiments, which yield the sample's real density profiles, were supplemented with classical MD simulations, which allows for a detailed study of surface density profiles as a function of distance from the interface. This combination of methods has been used successfully before, ${ }^{16-18}$ as their results reveal complementary information of the studied systems. Here, the surface thickness, which was determined from MD simulated density profiles, is combined with relative intensities from XPS experiments on a liquid micro-jet to quantify the surface enrichment of succinic acid in aqueous solution. For the purpose of connecting the microscopic composition of the surface to macroscopic properties of aqueous systems, the excess of succinic acid in the surface region was used to model surface tension of aqueous solutions using two different models. The modeled surface tension was compared to measured surface tension reported elsewhere. ${ }^{4}$

\section{Methods}

\subsection{XPS experiments}

The XPS measurements were performed at the Swedish national synchrotron facility MAX IV Laboratory, Lund University, at the soft X-ray beamline I411. Only a brief overview about the experimental setup is given here, details can be found elsewhere. ${ }^{19}$ In this setup, the sample solution is pushed through a glass nozzle, with an inner diameter of about $20 \mu \mathrm{m}$, into the experimental chamber, which is kept under vacuum. The synchrotron radiation intersects perpendicular to both the flow direction of the liquid micro-jet and the central axis of the hemispherical electron analyzer, which is mounted at $54.7^{\circ}$ (the so-called magic angle ${ }^{20}$ ) relative to the polarization plane of the synchrotron radiation. This geometry minimizes anisotropy effects in the resulting photoelectron spectra. ${ }^{15}$ The emitted photoelectrons exit the interaction zone through a skimmer, which is situated approximately $2 \mathrm{~mm}$ from the liquid surface. Subsequently, their kinetic energy is determined by a hemispherical electron energy analyzer (VG Scienta, R4000). Measurements are performed at a distance of 2-3 mm downstream from the nozzle, well before the micro-jet breaks up into droplets. After the interaction with X-ray radiation, a liquid nitrogen-cooled trap catches the remainders of the jet. The temperature of the liquid micro-jet at the interaction point is not known exactly. Before entering vacuum it was kept at stable temperature of about $10^{\circ} \mathrm{C}$, while evaporative cooling may come into play as the jet travels through the evacuated chamber. $^{21}$

A stock solution of $\mathrm{SuccH}_{2}$ was prepared freshly from commercially available chemicals (Sigma-Aldrich) and demineralized water $(18.2 \mathrm{M} \Omega \cdot \mathrm{cm}$, Millipore Direct-Q) for each of the two sets of measurements. The concentrations of the sample solutions are given in $\mathrm{mol} / \mathrm{dm}^{3}$, which is denoted with $\mathrm{M}$. The stock solutions ( 0.4 and $0.5 \mathrm{M}$, respectively) were filtered (Whatman Puradisc FP30 syringe filters, $1.2 \mu \mathrm{m}$ ) before dilution to remove solid particles, which may disturb the flow of the liquid jet and cause the injection system to fail. By dilution of the stock solution, the following concentrations were made available for the first series: $0.1,0.17,0.2,0.3$ and $0.4 \mathrm{M}$, and for the second series: $0.05,0.1$ and $0.5 \mathrm{M}$, respectively. Sodium hydroxide $(\mathrm{NaOH})$ was used to adjust $\mathrm{pH}$ for the investigation of succinate ions $\left(\mathrm{Succ}^{2-}\right)$ in aqueous solution. The following concentrations were prepared: $0.1,0.3,0.4 \mathrm{M}$ and $0.05,0.1$, $0.5 \mathrm{M}$, respectively. Furthermore, the bulk density of all studied solutions was measured using a density meter (DMA Anton paar). It was found that it deviates with about $1.5 \%$ for the highest concentration $(0.5 \mathrm{M})$ from that of pure water. The molar concentrations used in the XPS measurements can therefore, with only minor deviations, be directly translated to molal concentrations for the comparison to other studies. 
In all experiments, the succinic acid abundance was monitored via its carbon $1 \mathrm{~s}(\mathrm{C} 1 \mathrm{~s})$ photoemission $(\mathrm{PE})$ lines at a photon energy of $360 \mathrm{eV}$, at which also the $1 b_{1}$ valence PE line of liquid water was recorded. The latter is a measure of the overlap of the X-ray beam with the liquid micro-jet and the acceptance of the electron energy analyzer. The same spectrum was used to calibrate the kinetic energy scale by aligning the $1 b_{1} \mathrm{PE}$ line of liquid water to $11.16 \mathrm{eV}^{22}$ At a photon energy of $360 \mathrm{eV}$, the kinetic energy of the emitted $\mathrm{C} 1 \mathrm{~s}$ electrons is between 65 to $70 \mathrm{eV}$, which is, according to the so-called universal curve, ${ }^{17,23}$ close to that corresponding to the minimum effective attenuation length (EAL) of photoelectrons in condensed matter. Due to the short EAL, estimated to 5-10 $\AA$ for a liquid micro-jet, these kind of measurements are very surface sensitive.

Generally, the observed PE intensity at a given kinetic energy of the photoelectrons is proportional to the concentration of a compound in the surface layer and the photoionization cross section of the given species, but exponentially attenuated along its path, which can be quantified by the photoelectron's EAL, Figure 1. Due to these dependencies, this spectroscopic method enables probing of the real interfacial distribution of compounds at the water-vapor interface directly with chemical sensitivity, while the absolute amount of a certain species in the surface region is generally not available from PE spectra. However, qualitative information can be obtained from ratios of PE intensities of the same element. We therefore relate the $\mathrm{C} 1 \mathrm{~s} \mathrm{PE}$ intensities of succinic acid from different solutions with varying $\mathrm{pH}$ values and concentrations to each other.

For the direct comparison of PE intensities of different solutions to each other, the spectra must be normalized in a way that accounts for the flux of the X-ray beam. Since the recorded PE intensity in these experiments depends strongly on the experimental alignment of light source, liquid micro-jet and electron energy analyzer entrance, which can change over time, PE intensities of different sample solutions can only be compared if the alignment is persistent during a measurement session. This means in practice that the samples must be measured in quick succession, with reference spectra recorded between sample acquisitions to verify constant alignment. As reference, spectra of a $50 \mathrm{mM} \mathrm{LiBr}$ aqueous solution were attained, where variations of the signal intensities were used for error bar estimation. For the direct comparison between the different measurement sessions, the 0.1 and $0.3 \mathrm{M} \mathrm{SuccH}_{2}$ solutions were repeatedly measured and the $\mathrm{C} 1 \mathrm{~s}$ PE intensity was scaled accordingly.

Curve fitting was carried out using the $\mathrm{SPANCF}^{24}$ fitting routine for IGOR Pro (WaveMetrics, Inc, Lake Oswego, OR, USA). The C1s PE lines of succinic acid were fitted using Voigt line shapes. For the final analysis only the total PE intensity of each $\mathrm{C}$ 1s spectrum was used. To ensure a consistent line profile throughout the fit, all samples of one series were fitted simultaneously, where the Lorentzian and Gaussian line widths, and relative binding energies were forced to take the same values, while intensities were free to vary.

\subsection{Classical molecular dynamics}

Molecular dynamics simulations of succinic acid and succinate ions dissolved in water were performed using the GROMACS molecular dynamics software ${ }^{25}$ with non-polarizable force fields. The $\mathrm{SuccH}_{2}$ or $\mathrm{Succ}^{2-}$ molecules were modeled using the OPLS-AA force field, ${ }^{26}$ and TIP4P ${ }^{27}$ was used for the water molecules. The simulations consisted of 2000 water molecules and 4, 11 or $18 \mathrm{SuccH}_{2}$ molecules or Succ ${ }^{2-}$ ions, which corresponds to $0.1,0.3$ and $0.5 \mathrm{M}$ aqueous solutions, respectively. For the systems containing succinate ions, water molecules were replaced with the adequate number of sodium ions to neutralize the system. All simulations were performed at $\mathrm{T}=278.15 \mathrm{~K}$, with the temperature controlled by the Bussi thermostat. ${ }^{28}$ Each system was initially equilibrated for $1 \mathrm{~ns}$ in a cubic simulation box, after which the $\mathrm{z}$-dimension of the box was elongated, resulting in a liquid slab configuration in the center of a $3.9 \times 3.9 \times 13.0 \mathrm{~nm}^{3}$ simulation box. Periodic boundary conditions were applied in all directions. The bond lengths of the $\mathrm{SuccH}_{2}$ or Succ ${ }^{2-}$ species were constrained using the LINCS algorithm, ${ }^{29}$ and water molecules were kept rigid (both bond lengths and angles were kept constant) with the SETTLE algorithm. ${ }^{30}$ The long-range part of the Coulombic interactions was treated by particle-mesh Ewald summation. ${ }^{31}$ The cut-off for van der Waals interactions was set to $1.1 \mathrm{~nm}$ and the simulation timestep was $1 \mathrm{fs}$.

Generally, MD simulated density profiles give information on density changes as a function of the distance from the interface and enable determination of the thickness of the surface region. However, the absolute density of a species may vary somewhat depending on the employed force fields, which is why we focus on qualitative changes for further discussion.

\subsection{Surface enrichment from MD and XPS results}

In this work, the aim is to give quantitative surface enrichment factors for succinic acid at the aqueous interface, which can be used to compute the increased concentration of a species in the surface region by multiplying the surface enrichment factors with given bulk concentrations. These surface enrichment factors have been derived in a similar way in a previous work by Prisle et al. ${ }^{12}$ (supplementary information). Briefly summarized, since the electron's EAL and photoionization crosssections are not accurately known, a simple model, that describes the solution as being divided into a surface and a bulk region, where the change between the phases can be described as a step-function (Figure 1, left side), was used to compute surface enrichment factors for different bulk concentrations. From XPS experiments, the recorded PE intensity of the surface active succinic acid was compared to that of the divalent succi- 


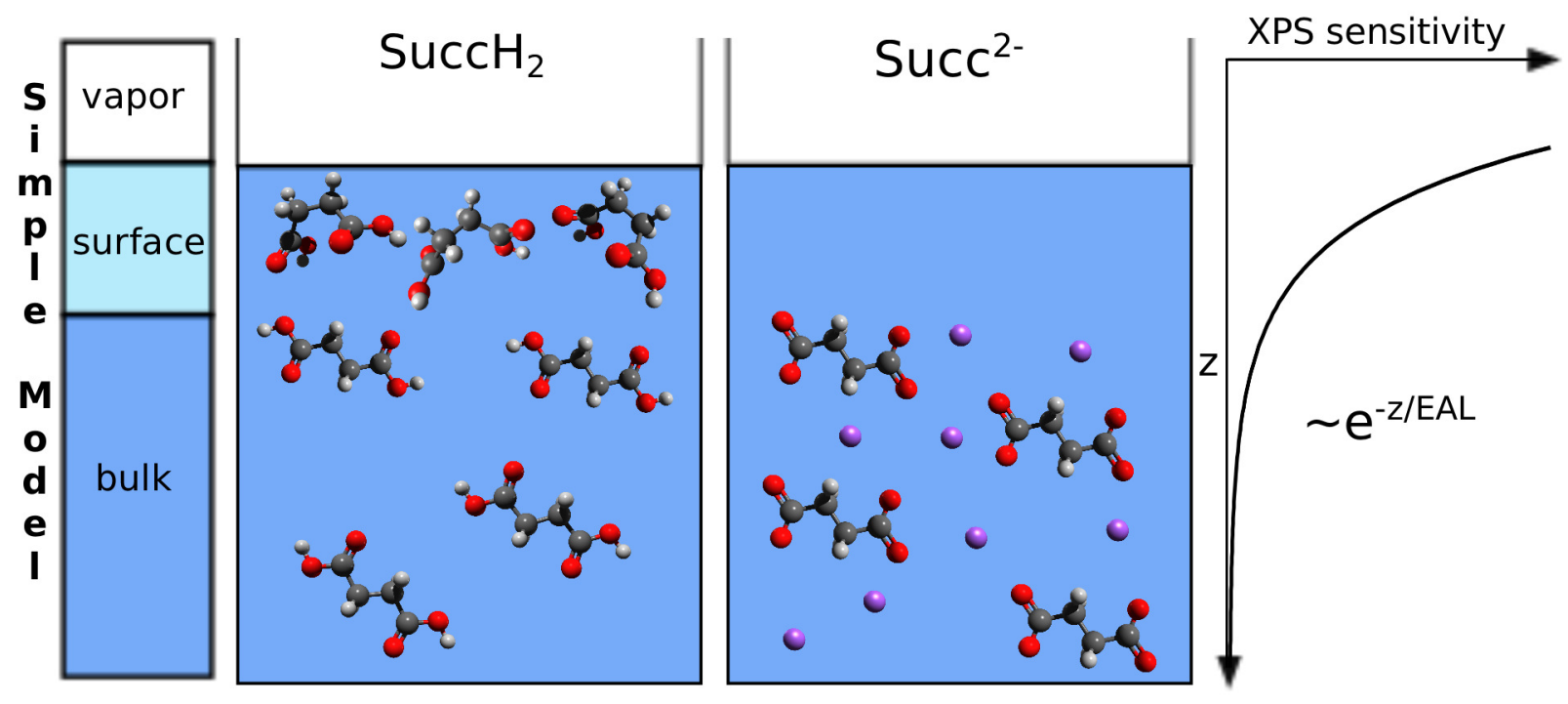

Fig. 1: Schematic of the simple model (left side) used to estimate surface enrichment factors for succinic acid solutions from known bulk concentrations (middle). Surface sensitive XPS experiments, which are used to investigate the surface concentration of surface active succinic acid in comparison to succinate ions, show that the two species exhibit different surface behaviors. The XPS sensitivity is exponentially attenuated along the path from the surface to the bulk of a solution (right side).

nate ion $\mathrm{Succ}^{2-}$, which turned out to avoid the surface region, see Figure 1. These findings were supplemented with results from MD simulations. The uncertainty of the probing depth in this experiment is described by a so-called sensitivity factor that gives the percentage of bulk and surface contribution to the total PE signal: $s=n_{B} / n_{S}$. A conservative estimate assumes a contribution of $50 \pm 25 \%$ of the total PE signal that comes from compounds in the surface region, ${ }^{23}$ which results in sensitivity factors in the range of $1 / 3<n_{B} / n_{S}<3$. Using this simple model, any PE signal ratio larger than 4 for the high bulk sensitivity (or larger than 1.33 for the low bulk sensitivity) indicates an enhanced surface concentration besides the higher propensity to reside at the aqueous surface, that is, a species' ability to be closer to the interface compared with another one. On the other hand, an observed PE signal ratio of less than 4 does not necessarily imply increased concentration in the surface but a possibly higher surface propensity of one studied species in comparison to another. Details on this estimation can be found in the electronic supplementary information $\left(\mathrm{ESI}^{\dagger}\right)$. In our final analysis, the broad range of possible sensitivity factors was narrowed with aid of MD simulated density profiles.

\section{Results and discussion}

\subsection{Probing the surface with XPS: Succinic acid at differ- ent protonation states}

Since succinic acid is a dicarboxylic acid, it has two carboxylic acid groups and thus three different possible protonation states. Acid-base-titration using a strong sodium hydroxide solution was performed to determine the $\mathrm{p} K_{\mathrm{a}}$ values for $0.3 \mathrm{M}$ succinic acid; details can be found in the $\mathrm{ESI}^{\dagger}$. The experimentally determined $\mathrm{p} K_{\mathrm{a}}$ values of succinic acid at $0.3 \mathrm{M}$ are 3.83 and 5.13. Below pH $=3.83$, the molecular form SuccH $_{2}\left(\mathrm{C}_{2} \mathrm{H}_{2}(\mathrm{COOH})_{2}\right)$ dominates; between $\mathrm{pH} 3.83$ and 5.13, the singly deprotonated form $\mathrm{SuccH}^{-}\left(\mathrm{C}_{2} \mathrm{H}_{2}(\mathrm{COO})_{2} \mathrm{H}^{-}\right)$dominates and above $\mathrm{pH} 5.13$, the doubly-deprotonated form Succ ${ }^{2-}\left(\mathrm{C}_{2} \mathrm{H}_{2}(\mathrm{COO})_{2}^{2-}\right)$ dominates in aqueous solution, see inset in Figure 2 (created using MEDUSA ${ }^{32}$ ), which shows the fraction of each protonation state of succinic acid as a function of $\mathrm{pH}$ at $0.3 \mathrm{M}$ total concentration. Note, SuccH ${ }^{-}$cannot be in aqueous solution as a pure species, since at the intermediate $\mathrm{pH}$ value, there is always a mixture of $\mathrm{SuccH}_{2}$, SuccH ${ }^{-}$and $\mathrm{Succ}^{2-}$ with a ratio of approximately 1:3:1 in the bulk of the aqueous solution.

$\mathrm{C} 1$ s core-level PE spectra, shown in Figure 2, were obtained from pure $0.3 \mathrm{M}$ succinic acid solutions at $360 \mathrm{eV}$ photon energy for the $\mathrm{pH}$ values 2.0 and 12.9 and the binding energies of $\mathrm{SuccH}_{2}$ and $\mathrm{Succ}^{2-}$ were determined. The PE intensity is displayed on an arbitrary scale as a function of binding energy of the $\mathrm{C} 1 \mathrm{~s}$ photoelectrons. Note that the relative PE intensity scales of the two different traces are the 


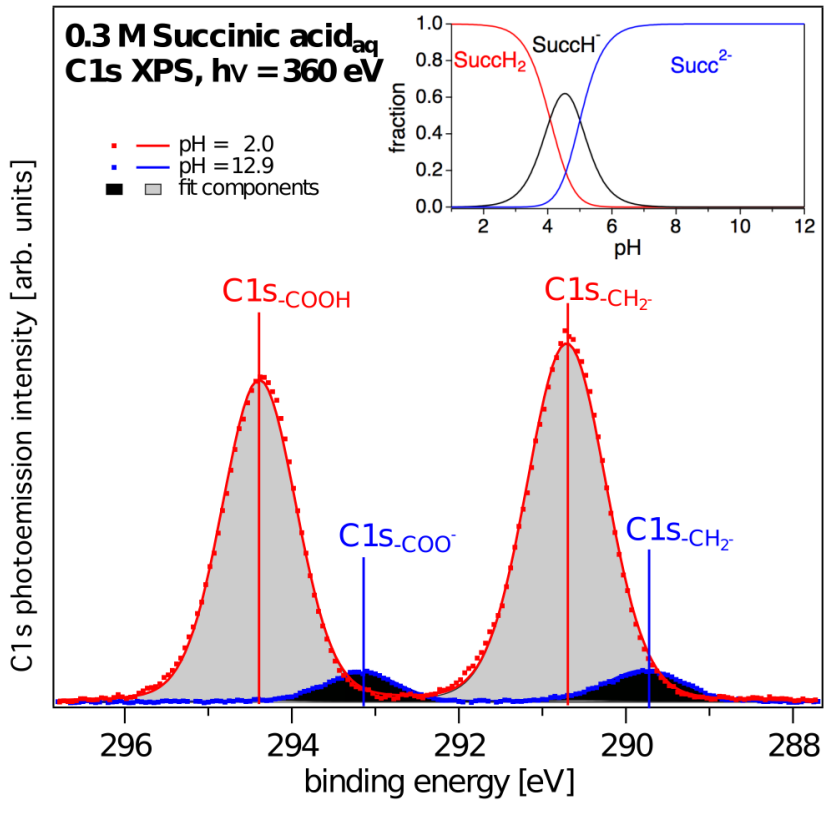

Fig. 2: C1s photoelectron spectra of $0.3 \mathrm{M}$ aqueous succinic acid at different $\mathrm{pH}$ values. Note that the relative intensity scale of the different traces is the same. Inset: Fraction of succinic acid form as a function of $\mathrm{pH}$ value created using MEDUSA. ${ }^{32}$

same. Due to the chemical shift of the C1s PE lines in different chemical environments, the distinction between signals from $-\mathrm{CH}_{2}$ - groups, protonated and deprotonated carboxylic acid/carboxylate groups can be done directly. Based on earlier results on mono-carboxylic acids and their salts, ${ }^{12,33}$ two $\mathrm{C} 1 \mathrm{~s} \mathrm{PE}$ lines are expected from succinic acid. The carboxylic $\mathrm{C} 1 \mathrm{~s} \mathrm{PE}$ line, originating from the two chemically equivalent carboxylic acid groups, has a higher binding energy than the $\mathrm{C} 1 \mathrm{~s} \mathrm{PE}$ line of the two equivalent $-\mathrm{CH}_{2}$ - groups. For succinate ions also two $\mathrm{C} 1 \mathrm{~s}$ PE lines are expected, both shifted towards lower binding energies.

With a full line width at half maximum (FWHM) of about $1.1 \mathrm{eV}$, the peaks in the PE spectrum are easily resolved, and the respective contributions of each form of succinic acid is determined, see Figure 2. Carboxylic acid C1s has highest binding energy, $294.4 \mathrm{eV}$, while the carboxylate $\mathrm{C} 1 \mathrm{~s}$ was found at slightly lower binding energy, $293.2 \mathrm{eV}$. Both have a higher binding energy than the $\mathrm{C} 1 \mathrm{~s}$ of the $-\mathrm{CH}_{2}$ - groups binding two carboxylic acid or two carboxylate groups, $290.7 \mathrm{eV}$ and $289.8 \mathrm{eV}$, respectively.

The PE intensity ratio of the $-\mathrm{CH}_{2}$ - to carboxylic/carboxylate $\mathrm{C} 1 \mathrm{~s}$ lines was found to be independent on the concentration and was fairly close to the stoichiometric ratio of 1:1 with $1.17 \pm$ 0.05 for $\mathrm{SuccH}_{2}$ and $\mathrm{Succ}^{2-}$. A specific orientation of the compounds at the surface cannot be stated only from this PE signal ratio. However, considering the constant ratio, no significant changes in orientation over the studied concentration range are anticipated. In recent publications by Blower et al. ${ }^{34}$ and Ruehl et al., ${ }^{35}$ where the orientation of succinic acid at the aqueous interface was investigated, supplemental evidence can be found. Their final analysis led to the conclusion that succinic acid in aqueous solution mainly orients with the carboxylic acid groups on the same side of the molecule when looking along its $\mathrm{CH}_{2}-\mathrm{CH}_{2}$-axis, which was also found by Roberts et al.. ${ }^{36}$ The orientation (cis-configuration), which is proposed in their works, was found to be most favorable for $\mathrm{SuccH}_{2}$ at the air/water interface, since both carboxylic acid groups can be fully hydrated while the alkyl backbone may be situated closer to or in the interface.

The ratio of the recorded C1s PE intensities of $\mathrm{SuccH}_{2}$ and Succ $^{2-}$ for the $0.3 \mathrm{M}$ solutions, Figure 2, was determined to be roughly 12 . This factor gives information on the relative propensity of a compound to reside in the surface region and its concentration. As described above, this high PE intensity ratio of more than 4 indicates a strong increase in concentration of $\mathrm{SuccH}_{2}$ in the surface region and this implies that $\mathrm{SuccH}_{2}$ features a higher propensity to reside in the surface region compared with Succ ${ }^{2-}$.

\subsection{Probing the surface with XPS: Succinic acid and suc- cinate ions at different concentrations}

To study the relative surface propensity and concentration as a function of the bulk concentration, a series of C1s PE spectra of succinic acid in aqueous solution were recorded over a concentration range of 0.05 to $0.5 \mathrm{M}$ at both low and high $\mathrm{pH}$ values, which are shown in Figures 3a) and b), respectively. The PE intensities are given in arbitrary units, but the relative intensity scale is the same for both and can be used for comparison.

It is immediately evident that the C1s PE intensity increases with increasing concentration in both cases. The fact that the $\mathrm{SuccH}_{2} \mathrm{C} 1 \mathrm{~s} \mathrm{PE}$ intensity is higher for all concentrations compared to Succ ${ }^{2-}$, indicates that the previously stated high surface propensity and increased surface concentration of succinic acid in aqueous solution is true over the full range of concentrations.

To quantify the change in PE intensity with increasing concentration, the recorded C1s PE intensities were obtained from the spectra in Figure 3 and plotted as a function of concentration in Figure 4. To provide visual clarity, the summed areas of both C1s PE lines were normalized to the C1s PE peak area of the $0.1 \mathrm{M} \mathrm{SuccH}_{2}$ solution. The PE intensities of the spectra of the divalent $\mathrm{Succ}^{2-}$, see Figure 3b), were normalized accordingly. The dashed straight lines in Figure 4 show how this PE intensity is expected to develop for a species that populates the surface region proportional to the bulk concentration, that is a distribution between surface and bulk that does not change with concentration. The error bars were estimated from variation in 
a)

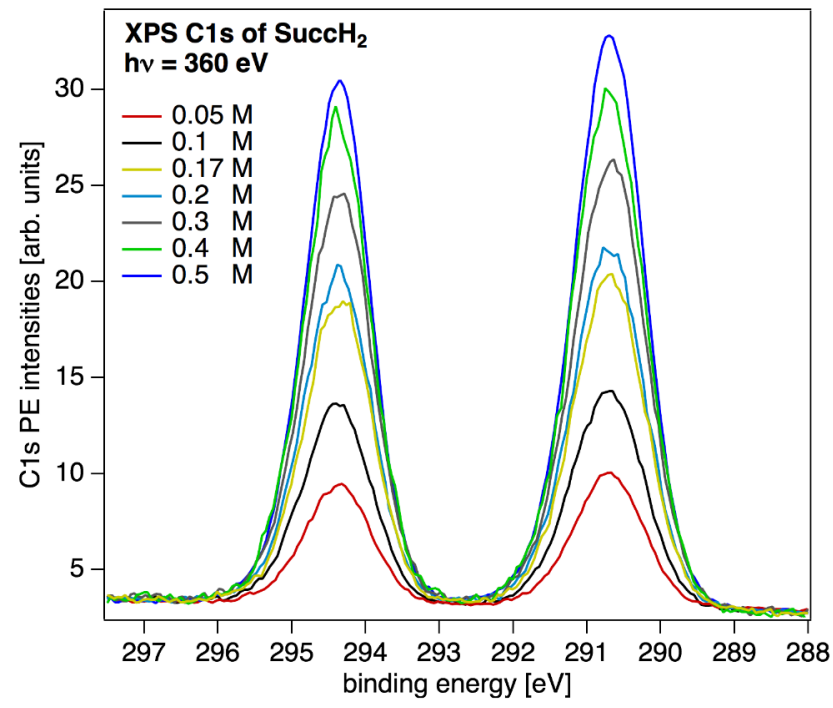

b)

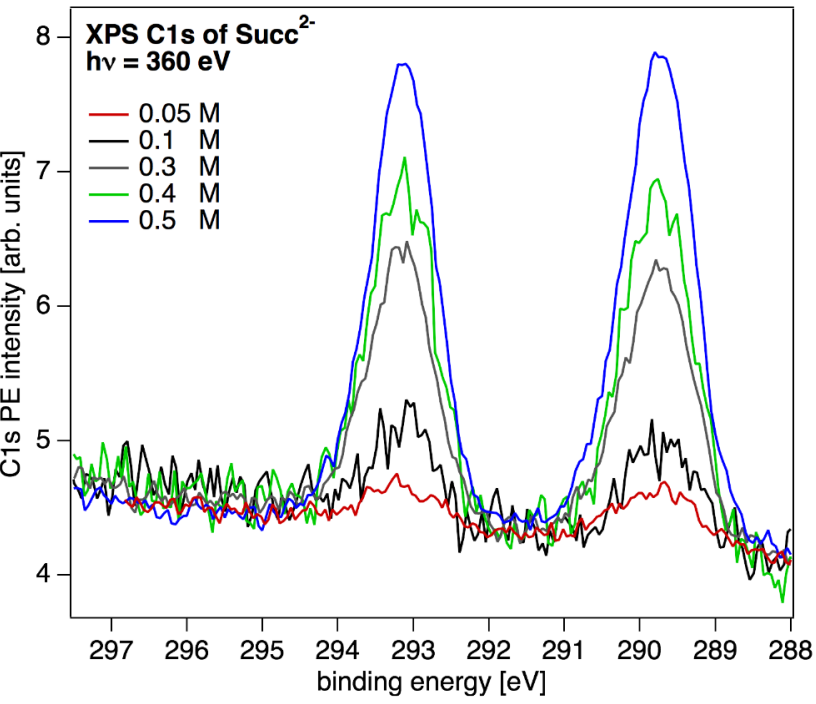

Fig. 3: $\mathrm{C} 1 \mathrm{~s} \mathrm{PE}$ spectra for aqueous succinic acid at low and high $\mathrm{pH}$ values: a) $\mathrm{SuccH}_{2}$ at $\mathrm{pH}=2$ at different concentrations. The PE intensity increases nonlinearly.; b) $\mathrm{PE} \mathrm{C1s} \mathrm{spectra} \mathrm{for} \mathrm{Succ}^{2-}$ at $\mathrm{pH}>12$ at different concentrations.

PE intensities and data fitting. Furthermore, the ratio was determined from the $\mathrm{C} 1 \mathrm{~s}$ PE intensities and surface enrichment factors were calculated for each concentration, which are listed in Table S2 in the ESI ${ }^{\dagger}$.

The curves in Figure 4 resemble the shape of Langmuir adsorption isotherms, ${ }^{37}$ which are used to describe the fraction of the surface area that is covered by adsorbents as a function of concentration of the solute. It can be seen that the PE intensity increases linearly with increasing concentration for the solutions with high $\mathrm{pH}$ value (see blue curve in Figure 4 ). This means that the amount of $\mathrm{Succ}^{2-}$ that is monitored in this experiment is increasing linearly with increasing bulk concentration, hence it resembles the bulk contribution of the recorded PE signal only. This is expected for Succ ${ }^{2-}$, since these strongly hydrated ions rather avoid the surface region due to the robust water shell around the carboxylate groups, which demand hydrogen bonding in three dimensions. The strength of hydration is related to the charge density of the solute. The higher charge density of the oxygen atoms in the carboxylate groups and its two charges, ${ }^{38}$ lead to the stronger hydrogen bonding network of succinate ions in comparison to, e.g., singly charged halide ions, such as iodide or bromide, which are known to be less depleted from the aqueous interface. This finding is supported by the fact that surface tension experiments of aqueous solutions containing succinate ions, show a linear increase in surface tension with increasing concentration, ${ }^{39}$ which generally suggests a depletion from the aqueous surface.

The $\mathrm{C} 1$ s PE intensity of $\mathrm{SuccH}_{2}$ is found to be much higher in comparison to the PE intensity of the $\mathrm{Succ}^{2-}$ and increases non-linearly with increasing concentration, where the PE signal changes less for higher than for lower concentrations. Within the experimental uncertainties, the amount of $\mathrm{SuccH}_{2}$ is only slightly increased from 0.4 to $0.5 \mathrm{M}$ concentration, Figure 4, which is very close to the solubility limit of $\mathrm{SuccH}_{2}$ in water. ${ }^{40}$ This implies that $\mathrm{SuccH}_{2}$ accumulates at the surface and at higher concentrations the surface region is very close to saturation. Increasingly more $\mathrm{SuccH}_{2}$ are located in the bulk region of the solution, because the number of available sites for $\mathrm{SuccH}_{2}$ molecules in the aqueous surface region gets fewer with increasing concentration and at the same time the number of water molecules available per molecule is gradually decreasing. Reduced surface tensions of succinic acid solutions compared with pure water supports these observations. ${ }^{4,6}$

At concentrations that are closer to the solubility limit, when many molecules disturb the hydrogen bonding network of water, it is easier for $\mathrm{SuccH}_{2}$ to also stay in the bulk. Another aspect of this interplay between solute and solvent is that fewer possibilities for the $\mathrm{SuccH}_{2}$ to reside in the surface region forces the water to break hydrogen bonds in bulk water to hydrate the acid molecules.

At lower concentrations the few molecules in the solution do not affect each other and a non-negligible portion of the $\mathrm{SuccH}_{2}$ can reside close to the interface, which is still only a very small percentage of all $\mathrm{SuccH}_{2}$ molecules in the sample. It is energetically more favorable for the whole system to place $\mathrm{SuccH}_{2}$ molecules in the interface. In that way less water molecules need to be situated at these energetically unfavorable surface sites and the strong hydrogen bonding network in the aqueous 


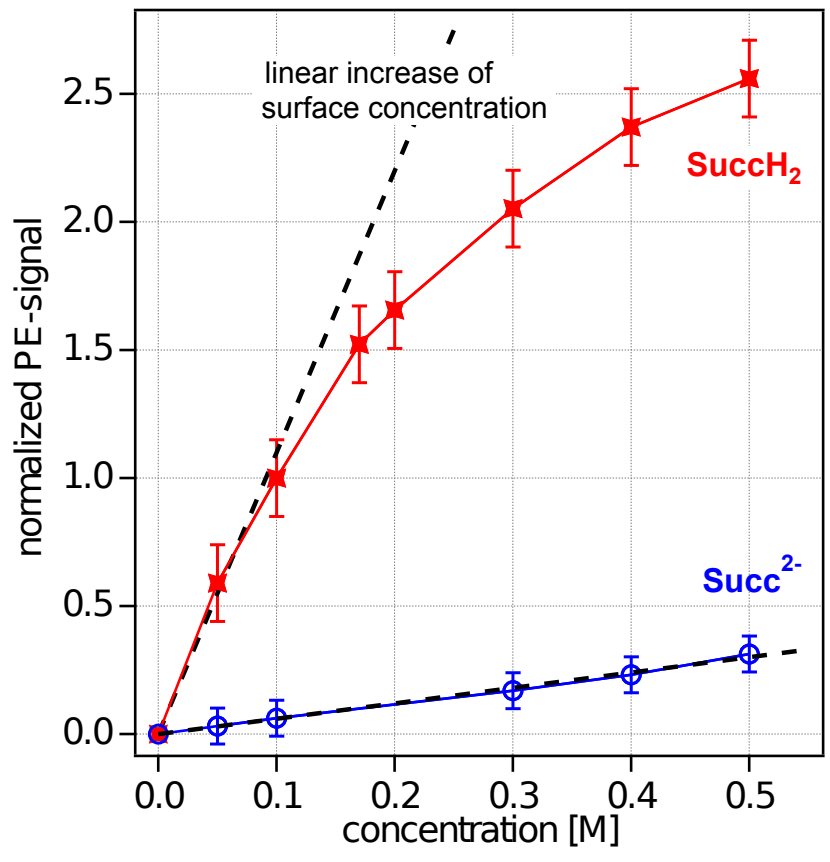

Fig. 4: $\mathrm{C} 1 \mathrm{~s} \mathrm{PE}$ intensities of succinic acid at different $\mathrm{pH}$ values, obtained from the spectra shown in Figures 3, as a function of bulk concentration. All intensities were normalized to the $\mathrm{C} 1 \mathrm{~s}$ PE lines area of the $0.1 \mathrm{M} \mathrm{SuccH}_{2}$ solution. The blue curve shows the normalized $\mathrm{PE}$ intensity of the solutions at $\mathrm{pH} 2$, while the green curve shows the normalized PE intensity of the solutions at $\mathrm{pH} 12$. The dashed lines show the hypothetical case of a constant surface increment with bulk concentration for $\mathrm{SuccH}_{2}$ at $\mathrm{pH} 2$.

bulk can be maintained to a larger extent.

Due to the much higher spectral intensities of $\mathrm{SuccH}_{2}$ compared to the $\mathrm{Succ}^{2-}$ over the whole concentration range it can be concluded that $\mathrm{SuccH}_{2}$ is strongly enriched in the surface region. While the neutral form of succinic acid can approach the aqueous interface closer due to its non-charged carboxylic groups and the aliphatic carbons, hydrophilic interactions of the charged carboxylate groups predominate over hydrophobic forces from the aliphatic carbons for succinate ions. Thus, the divalent anions, which generally interact strongly with the surrounding water molecules, mainly stay in the bulk of the solution, where full hydration can be achieved. ${ }^{16,41}$

\subsection{Simulating the surface with MD}

Density profiles from MD simulations are shown as a function of distance from the slab center in Figure 5. For visual clarity, the water oxygen profile is scaled by the density value at the center of the slab, and the various $\mathrm{SuccH}_{2}$ and $\mathrm{Succ}^{2-}$ profiles are normalized so that their integral value is the same. Figures 5a) and 5b) show the carbon profiles of $\mathrm{SuccH}_{2}$ and $\mathrm{Succ}^{2-}$, respectively, for all studied concentrations. It can easily be seen that the molecular form $\mathrm{SuccH}_{2}$ is strongly enhanced in the surface region, in agreement with previous MD results on aqueous solutions of dicarboxylic acids. ${ }^{5,42}$ On the other hand, Succ $^{2-}$ clearly prefers to stay in the bulk as the density drops to effectively zero already before the surface region. This result is in line with the findings from the XPS experiments, stating a strong propensity of $\mathrm{SuccH}_{2}$ molecules to reside at the aqueous surface, while Succ ${ }^{2-}$ ions are strongly depleted, as is depicted in the schematics in Figure 1.
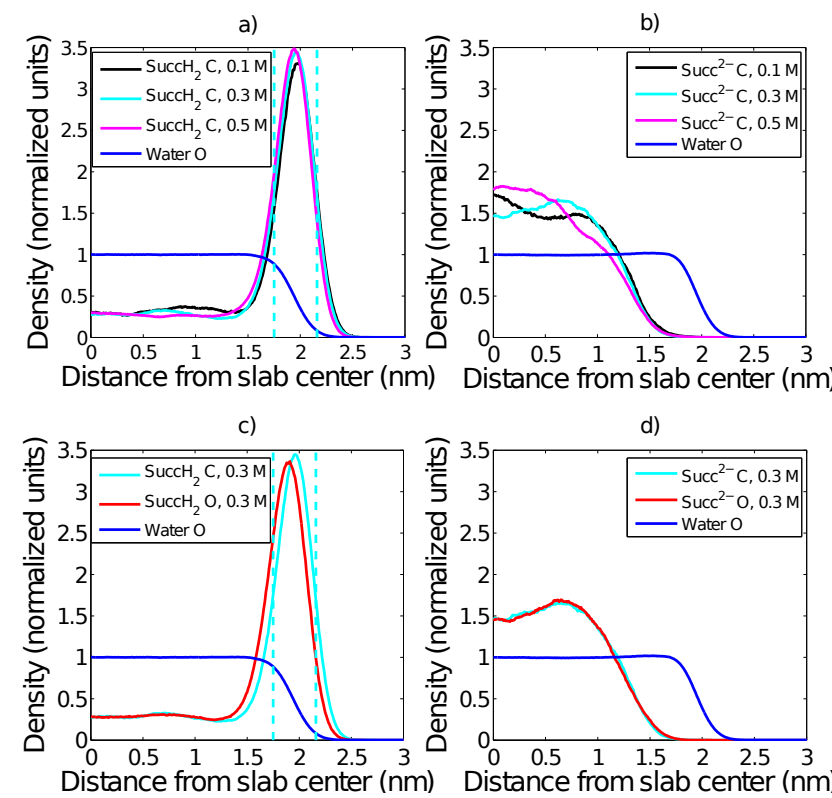

Fig. 5: Simulated carbon density profiles for a) $\mathrm{SuccH}_{2}$ and b) Succ ${ }^{2-}$ in aqueous solution at various concentrations. The simulated oxygen density profiles are shown together with the respective carbon densities in c) and d). The vertical lines in a) and c) show the full width half maximum for the corresponding carbon profile. It can be seen that the neutral form is clearly enriched at the water/vapor interface, while the doubly charged form is strongly depleted.

Figures 5c) and d) show the carbon and oxygen profiles of $0.3 \mathrm{M} \mathrm{SuccH}_{2}$ and $\mathrm{Succ}^{2-}$, respectively. While the carbon profiles for the different concentrations are similar to each other, the oxygen profile of $\mathrm{SuccH}_{2}$ is slightly shifted towards the bulk and away from the aqueous interface, see Figure 5c). The oxygen profiles for 0.1 and $0.5 \mathrm{M}$ concentrations (not shown), show the same difference between the carbon and oxygen profiles. This supports the previously mentioned preferential orientation of the molecule. Due to stronger hydration, the carboxylic acid groups point towards the bulk region, while the aliphatic carbons in the middle can approach the surface more closely, as depicted in Figure 1. For the divalent anion, see Figure 5d), the carbon and the oxygen density profiles match with only minor deviations, which can be interpreted as a random orientation in the bulk solution. 
In order to quantify the excess amount of succinic acid in the surface region compared to the known bulk concentration, a surface enrichment factor can be calculated directly from the obtained density profiles by computing the ratio of the carbon density of $\mathrm{SuccH}_{2}$ in the surface and its density in the bulk. However, a definition for the location of the surface is required.

A surface definition that matches the rectangular surface phase form of the surface-bulk-model, that was introduced earlier (Figure 1), can be applied here. The FWHM of the carbon profile peak $D$ yielding $4 \AA$, is applied as limits for the surface region, which are shown as vertical lines for $0.3 \mathrm{M} \mathrm{SuccH}_{2}$ in Figure 5a). Hence, a molecule is considered to reside in the bulk of the solution or in the surface region based on where the center of mass of the molecule is located. Adopting this surface definition yields largely constant surface enrichment factors around 14 to 15 over the whole concentration range. The magnitude of the MD surface enrichment factors is in good agreement with the surface enrichment factors obtained from results of the XPS experiments, Table S2 $\mathrm{ESI}^{\dagger}$.

Furthermore, this surface definition is used to give an improved estimate of the surface sensitivity factor $n_{B} / n_{S}$ introduced above, that is mainly depending on the electron's EAL and the thickness of the surface, see $\mathrm{ESI}^{\dagger}$ for details. The previously given conservative estimate of bulk contribution to the PE signal of 25 to $75 \%$ is significantly narrowed to a range between 45 to $67 \%$ bulk contribution, where the remaining uncertainty for the sensitivity factor is caused by the lack of exact knowledge of the electron's EAL from a liquid micro-jet at present. If the exact EAL was known, the enrichment factor of succinic acid as a function of concentration of the bulk solution was readily available from the XPS experiment.

It can be concluded that the combination of MD simulation results with the results from XPS experiments enables the derivation of the concentration of succinic acid in the surface layer as multiple of the known bulk concentrations by using ratios of $\mathrm{C} 1 \mathrm{~s}$ PE intensities of succinic acid and succinate ions, respectively.

\section{Connection to macroscopic surface tension}

The surface composition and concentration are key factors determining the surface tension of a given mixture. We use the surface enrichment factors of succinic acid, that were derived with aid of MD simulations and XPS experiments, as input for two different thermodynamic models to derive surface tensions of binary solutions of succinic acid in water. This gives further insight into the microscopic origin and role of surface enrichment on the surface tension of succinic acid in aqueous solution.

\subsection{Surface tension models}

Several methods are available to model the surface tension of aqueous solutions. Some of them are semi-empirical like the Szyszkowski equation ${ }^{43}$ and the Szyszkowski-Langmuir relation, ${ }^{44}$ while others describe surface-containing systems based on thermodynamic principles. Here two alternative thermodynamic models are tested to estimate the surface tension of aqueous succinic acid solutions: 1) a model introduced by Sprow and Prausnitz ${ }^{45,46}$ and later used by, e.g., Li et al. ${ }^{47}$ for aqueous electrolyte solutions; and 2) a simple mixture model where the surface tension of the solution is a mole-fraction weighted average of the pure compound's surface tensions. In both models the aqueous system is assumed to consist of three phases: vapor, surface and bulk, similar to the model introduced in Figure 1.

In the approach applied by $\mathrm{Li}$ et al. ${ }^{47}$ the surface and bulk layers are assumed to be in thermodynamic equilibrium, and the specific molar surface area of water at the surface phase is assumed to be equal to that of the bulk phase. With these assumptions, the surface tension of the aqueous solution $\sigma_{\text {sol }}$ can be approximated with

$$
\sigma_{s o l}=\sigma_{w}+\frac{R T}{A_{w}} \ln \frac{a_{w}^{S}}{a_{w}^{B}}
$$

where $\sigma_{w}$ is the surface tension of pure water, $R$ is the universal gas constant, $T$ is absolute temperature of the system and $A_{w}$ is the molar surface area of water which is defined as: ${ }^{47}$

$$
A_{w}:=\left(V_{w}\right)^{2 / 3}\left(N_{A}\right)^{1 / 3}
$$

where $N_{A}$ is Avogadro's constant and $V_{w}$ the molar volume of pure water which is equal to the quotient of the molar mass of water and its density $M_{w} / \rho_{w} . a_{w}^{S}$ and $a_{w}^{B}$ are the activities of water in the surface and bulk phases, respectively. Activity of water is equal to $a_{w}=x_{w} \cdot \gamma_{w}$, in which $\gamma_{w}$ is the activity coefficient and $x_{w}$ is the mole fraction of water. The activity was calculated separately for the bulk solution using $x_{w}^{B}$ and $\gamma_{w}^{B}$ and for the surface layer $x_{w}^{S}$ and $\gamma_{w}^{S}$. The mole fraction of water $x_{w}^{S}$ in the surface was calculated based on the molality concentration of the organic in the surface $m_{\text {org }}^{S}$, which is in turn obtained as a multiple of the molality concentration of the organic in the bulk $m_{\text {org }}^{B} 47$

$$
m_{\text {org }}^{S}=g \cdot m_{\text {org }}^{B}
$$

where $g$ is the surface enrichment factor. The concentrations of the organic acid in the surface and in the bulk phase are given in mol/kg water. The activity coefficients $\gamma_{w}^{S}$ and $\gamma_{w}^{B}$ of water in the mixture are calculated using the UNIFAC model. ${ }^{48,49}$ The surface tension of pure water at $10^{\circ} \mathrm{C}$ given by Vanhanen et al. ${ }^{4}$ as $73.8 \mathrm{mN} / \mathrm{m}$ is used. 
In the simple mixture model the surface tension of the solution $\sigma_{\text {sol }}$ is expressed as a linear combination of the pure compound's surface tension $\sigma_{\text {org }}$ weighted with its mole fraction in the surface layer $x_{\text {org }}^{S}$ and the surface tension of pure water $\sigma_{w}{ }^{50}$

$$
\sigma_{\text {sol }}=\left(1-x_{\text {org }}^{S}\right) \sigma_{w}+x_{o r g}^{S} \sigma_{o r g}
$$

We are now in a position to compare the surface tension values obtained by using the surface enrichment factors obtained from XPS experiments and MD simulations in the two models with experimentally obtained surface tension values from Vanhanen et al. ${ }^{4}$ at $10^{\circ} \mathrm{C}$, which is the temperature closest to that of the liquid micro-jet used in the XPS experiment where surface tension observations were available in literature.

\subsection{Correlation of experimental to modeled surface ten- sions using surface enrichment factors}

In Figures 6a) and b), the calculated and experimental surface tension values obtained from the two different models, respectively, are plotted as a function of concentration. As the bulk contribution to the obtained PE intensities in XPS experiments is not known exactly, the surface tension as a function of concentration can follow different trend lines. The trend lines for the conservative estimate and the trend lines obtained by using the estimated surface thickness $\mathrm{D}=4 \AA$ from MD density profiles and physically reasonable values for the electron's EAL on liquid micro-jets ( 5-10 $\AA$ ) are shown. The surface enrichment factors $g$ obtained for the different bulk concentrations are listed in Table S2 in the $\mathrm{ESI}^{\dagger}$.

The figures show i) the surface tension for pure water; ii) surface tension calculated with enrichment factors from XPS experiments using the conservative estimate for the sensitivity considering $25 \%, 50 \%$ and $75 \%$ of the total PE signal coming from the bulk of the solution; iii) surface tension derived with enrichment factors from the XPS experiment and MD simulation results (45\% and 67\%); and iv) the experimentally obtained surface tension values from Vanhanen et al. at $10^{\circ} \mathrm{C} .{ }^{4}$ Note, higher bulk contribution leads to lower modeled surface tensions.

Surface enrichment factors that result in a best fit to the experimental surface tension data for the model introduced by Sprow and Prausnitz are in the range $g=19-24$. For the simple mixture model a range of $g=29-35$ is required to reproduce the measured surface tension data. Modeled surface tension values using enrichment factors of succinic acid obtained from MD and XPS experiments reproduce the measured surface tension values of Vanhanen et al. ${ }^{4}$ with only minor deviations at higher concentrations. Values derived from the simple mixture model show larger deviations from the measured surface tension values. The Sprow and Prausnitz model gives good correlations between modeled surface tensions with input data from XPS and MD simulations and experimentally obtained surface tensions by Vanhanen et al. ${ }^{4}$ This shows the direct correlation between increased surface concentration of succinic acid on the molecular level and surface tension, which is a macroscopic property of an aqueous solution.

\section{Conclusions}

Both results from XPS experiments and MD simulation suggest increased propensity of succinic acid to reside at the aqueous interface over the whole concentration range studied, while succinate ions avoid the surface region. The observed behaviors can be understood considering the strong hydration of the carboxylate groups, which coordinate water molecules around them in three dimensions. The less strongly hydrated carboxylic acid groups and the weakly hydrated aliphatic carbon atoms allow the succinic acid molecules to come closer to the aqueous surface than its ionic form, which is the basis of the relatively high surface propensity of succinic acid.

Moreover, succinic acid was found to have a higher concentration in the surface region compared to the bulk concentration, which was quantified in this work by the determination of surface enrichment factors. Concentration dependent PE spectra show that the excess of succinic acid in the surface is less strong at high bulk concentrations compared with low bulk concentrations. It was found that succinic acid saturates the aqueous surface at increased concentrations.

Results from MD simulations were used to estimate the surface layer thickness, which enables us to quantify the surface sensitivity of XPS experiments on liquid micro-jets more closely. The combination of the two techniques allows the derivation of the surface concentration of succinic acid as a multiple of the known bulk concentration by careful analysis of C1s PE intensities. The obtained surface concentrations were used to model surface tension using two different models. The derived surface tensions from the Sprow and Prausnitz model reproduce the experimentally obtained surface tension data from Vanhanen et al. with only minor deviations at higher concentrations, while the simple mixture model requires much higher surface enrichment factors to reproduce the same data. The comparison with surface tension values elucidates the direct connection between the increased surface concentration compared with bulk concentration and the reduced surface tension of aqueous solutions of succinic acid.

For atmospheric aerosol particles, which have a high surface to bulk ratio, surface phenomena are exceedingly important. Thus, surface to bulk partitioning as it has been studied in this work becomes even more significant especially for very small systems and may alter the particles surface properties. ${ }^{8}$

As particles in the atmosphere usually consist as mixtures of organic and inorganic compounds, further studies on the molecular properties of these surfaces are warranted. ${ }^{10}$ The perfor- 
a) Sprow and Prausnitz model

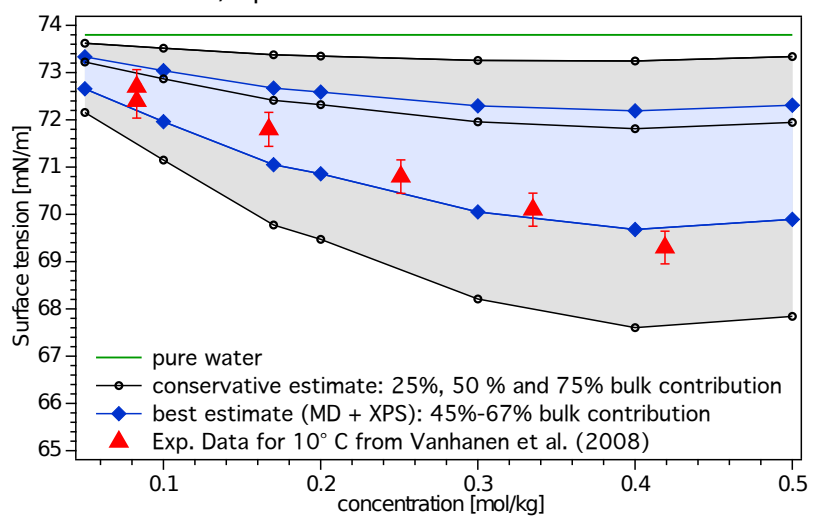

b) Simple mixture model

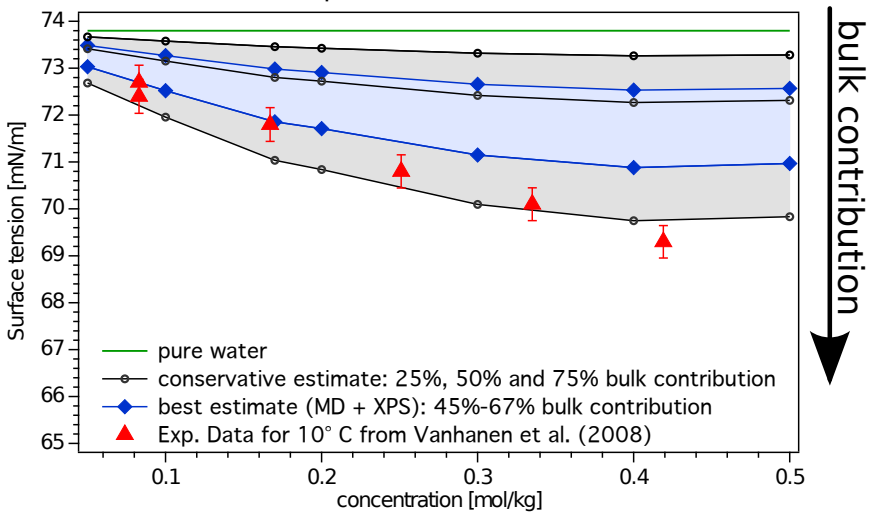

Fig. 6: The impact of surface enrichment on calculated surface tensions and their comparison to experimental data at $10^{\circ} \mathrm{C}$. Red symbols: Experimental data from Vanhanen et al., Ref. 4. Blue lines: surface tension of aqueous solutions of succinic acid calculated with: a) the model by Sprow and Prausnitz, Refs. 45-47, see Eq. 1; b) with the simple mixture model see Eq. 4, and surface enrichment factors derived from XPS experiments and MD simulations. Black lines: surface tension of aqueous solutions of succinic acid calculated using the conservative estimate of enrichment factors. The green curve corresponds to the surface tension of pure water at $10^{\circ} \mathrm{C}$.

mance of thermodynamic models in describing the behavior of mixed systems strongly depends on the specific mixture. ${ }^{51}$ Experimental input, as it could be provided from similar experiments on ternary solutions in the future, would thus be helpful in producing such information and furthermore constraining theoretical approaches for modeling these systems.

\section{Acknowledgments}

Financial support is gratefully acknowledged from the Swedish Research Council (VR, grants no. 2010-4140 and 2011-5120), the European Research Council (ERC-StG-ATMOGAIN, grant no. 278277) and the Knut and Alice Wallenbergs foundation. The authors would like to thank the staff at the MAX IV Laboratory and beamline I411 for smooth operation of the synchrotron ring and experimental facility.

\section{References}

1 D. Rosenfeld, U. Lohmann, G. B. Raga, C. D. O’Dowd, M. Kulmala, S. Fuzzi, A. Reissell and M. O. Andreae, Science, 2008, 321, 1309-1313.

2 M. Hallquist, J. C. Wenger, U. Baltensperger, Y. Rudich, D. Simpson, M. Claeys, J. Dommen, N. M. Donahue, C. George, A. H. Goldstein, J. F. Hamilton, H. Herrmann, T. Hoffmann, Y. Iinuma, M. Jang, M. E. Jenkin, J. L. Jimenez, A. Kiendler-Scharr, W. Maenhaut, G. McFiggans, T. F. Mentel, A. Monod, A. S. H. Prévôt, J. H. Seinfeld, J. D. Surratt, R. Szmigielski and J. Wildt, Atmos. Chem. Phys., 2009, 9, 5155-5236.

3 I. Riipinen, I. K. Koponen, G. P. Frank, A.-P. Hyvärinen, J. Vanhanen, H. Lihavainen, K. E. J. Lehtinen, M. Bilde and M. Kulmala, J. Phys. Chem. A, 2007, 111, 12995-13002.

4 J. Vanhanen, A.-P. Hyvärinen, T. Anttila, T. Raatikainen, Y. Viisanen and H. Lihavainen, Atmos. Chem. Phys., 2008, 8, 4595-4604.
5 S. Mahiuddin, B. Minofar, J. M. Borah, M. R. Das and P. Jungwirth, Chem. Phys. Lett., 2008, 462, $217-221$.

6 A.-P. Hyvärinen, H. Lihavainen, A. Gaman, L. Vairila, H. Ojala, M. Kulmala and Y. Viisanen, J. Chem. Eng. Data, 2006, 51, 255-260.

7 K. Salo, Å. M. Jonsson, P. U. Andersson and M. Hallquist, J. Phys. Chem. A, 2010, 114, 4586-4594.

8 R. Sorjamaa, B. Svenningsson, T. Raatikainen, S. Henning, M. Bilde and A. Laaksonen, Atmos. Chem. Phys., 2004, 4, 2107-2117.

9 N. Prisle, T. Raatikainen, R. Sorjamaa, B. Svenningsson, A. Laaksonen and M. Bilde, Tellus B, 2011, 60, 416-431.

10 N. L. Prisle, T. Raatikainen, A. Laaksonen and M. Bilde, Atmos. Chem. Phys., 2010, 10, 5663-5683.

11 P. Atkins, V. Walters and J. De Paula, Physical Chemistry, Macmillan Higher Education, 2006.

12 N. L. Prisle, N. Ottosson, G. Öhrwall, J. Söderström, M. Dal Maso and O. Björneholm, Atmos. Chem. Phys., 2012, 12, 12227-12242.

13 K. C. Duffey, O. Shih, N. L. Wong, W. S. Drisdell, R. J. Saykally and R. C. Cohen, Phys. Chem. Chem. Phys., 2013, 15, 11634-11639.

14 R. Seidel, S. Thürmer and B. Winter, J. Phys. Chem. Lett., 2011, 2, 633 641.

15 S. Hüfner, Photoelectron Spectroscopy: Principles and Applications, Springer-Verlag Berlin Heidelberg, 1995.

16 N. Ottosson, A. O. Romanova, J. Söderström, O. Björneholm, G. Öhrwall and M. V. Fedorov, J. Phys. Chem. B, 2012, 116, 13017-13023.

17 N. Ottosson, M. Faubel, S. E. Bradforth, P. Jungwirth and B. Winter, J. Electron Spectrosc., 2010, 177, 60-70.

18 J. Werner, E. Wernersson, V. Ekholm, N. Ottosson, G. Öhrwall, J. Heyda, I. Persson, J. Söderström, P. Jungwirth and O. Björneholm, J. Phys. Chem. B, 2014, 118, 7119-7127. 
19 H. Bergersen, R. R. T. Marinho, W. Pokapanich, A. Lindblad, O. Björneholm, L. J. Sæthre and G. Öhrwall, J. Phys.: Condens. Matter, 2007, 19, 326101-326110.

20 J. Cooper and R. N. Zare, J. Chem. Phys, 1968, 48, 942-943.

21 M. Faubel, K. R. Siefermann, Y. Liu and B. Abel, Acc. Chem. Res., 2012, 45, 120-130.

22 B. Winter, R. Weber, W. Widdra, M. Dittmar, M. Faubel and I. Hertel, J. Phys. Chem. A, 2004, 108, 2625-2632.

23 C. Powell and A. Jablonski, Nucl. Instrum. Methods, A, 2009, 601, 54-65.

24 Spectrum Analysis by Curve Fitting (SPANCF) macro package for Igor Pro by Edwin Kukk (edwin.kukk@utu.fi), University of Turku, Finland..

25 B. Hess, C. Kutzner, D. van der Spoel and E. Lindahl, J. Chem. Theory Comput., 2008, 4, 435-447.

26 W. L. Jorgensen, D. S. Maxwell and J. Tirado-Rives, J. Am. Chem. Soc., 1996, 118, 11225-11236.

27 W. L. Jorgensen, J. Chandrasekhar, J. D. Madura, R. W. Impey and M. L. Klein, J. Chem. Phys., 1983, 79, 926-935.

28 G. Bussi, D. Donadio and M. Parrinello, J. Chem. Phys., 2007, 126, 1410114107.

29 B. Hess, C. Kutzner, D. van der Spoel and E. Lindahl, J. Chem. Theory Comput., 2008, 4, 435-447.

30 S. Miyamoto and P. A. Kollman, J. Comput. Chem., 1992, 13, 952-962.

31 U. Essmann, L. Perera, M. L. Berkowitz, T. Darden, H. Lee and L. G. Pedersen, J. Chem. Phys., 1995, 103, 8577-8593.

32 I. Puigdomenech, Chemical Equilibrium Diagrams, http: / / www . kth. se/che/medusa, Accessed: March 2014.

33 N. Ottosson, E. Wernersson, J. Söderström, W. Pokapanich, S. Kaufmann, S. Svensson, I. Persson, G. Öhrwall and O. Björneholm, Phys. Chem. Chem. Phys., 2011, 13, 12261-12267.

34 P. G. Blower, S. T. Ota, N. A. Valley, S. R. Wood and G. L. Richmond, J. Phys. Chem. A, 2013, 117, 7887-7903.

35 C. R. Ruehl and K. R. Wilson, J. Phys. Chem. A, 2014, 118, 3952-3966.

36 J. D. Roberts, Acc. Chem. Res., 2006, 39, 889-896.

37 A. Adamson and A. Gast, Physical Chemistry of Surfaces, Wiley, 1997.

38 B. Roux, H. A. Yu and M. Karplus, J. Phys. Chem., 1990, 94, 4683-4688.

39 R. Taft and F. H. Welch, Trans Kans Acad Sci, 1951, 54, pp. 233-246.

40 M. Davies and D. M. L. Griffiths, Trans. Faraday Soc., 1953, 49, 1405 1410 .

41 B. Minofar, M. Mucha, P. Jungwirth, X. Yang, Y.-J. Fu, X.-B. Wang and L.-S. Wang, J. Am. Chem. Soc., 2004, 126, 11691-11698.

42 X. Ma, P. Chakraborty, B. J. Henz and M. R. Zachariah, Phys. Chem. Chem. Phys., 2011, 13, 9374-9384.

43 B. Szyszkowski, Z. Phys. Chem., 1908, 64, 385-414.

44 I. Langmuir, J. Am. Chem. Soc., 1917, 39, 1848-1906.
45 F. B. Sprow and J. M. Prausnitz, Trans. Faraday Soc., 1966, 62, 10971104.

46 F. B. Sprow and J. M. Prausnitz, Trans. Faraday Soc., 1966, 62, 11051111.

47 Z.-B. Li, Y.-G. Li and J.-F. Lu, Ind. Eng. Chem. Res., 1999, 38, 1133-1139.

48 A. Fredenslund, R. L. Jones and J. M. Prausnitz, AIChE Journal, 1975, 21, 1086-1099.

49 T. Raatikainen and A. Laaksonen, Atmos. Chem. Phys., 2005, 5, 24752495.

50 B. Poling, J. Prausnitz and J. Connell, The Properties of Gases and Liquids, McGraw-Hill Education, 2000.

51 H. Henschel, J. C. A. Navarro, T. Yli-Juuti, O. Kupiainen-Määttä, T. Olenius, I. K. Ortega, S. L. Clegg, T. Kurtén, I. Riipinen and H. Vehkamäki, J Phys. Chem. A, 2014, 118, 2599-2611. 